\title{
O Experimento de Hardy: a mais simples prova da violação do realismo local EPR
}

\author{
Hardy's Experiment: the simplest proof of the violation of EPR's Local Realism
}

\author{
Lucio S. Fassarella*10
}

${ }^{1}$ Universidade Federal do Espírito Santo, Departamento de Matemática Aplicada, Rodovia Governador Mário Covas, Km 60 Bairro Litorâneo CEUNES, São Mateus, ES, Brasil

Recebido em 05 de Abril, 2019. Aceito em 07 de Maio, 2019

\begin{abstract}
O Experimento de Hardy constitui um tipo de experimento físico pelo qual podemos demonstrar e verificar a validade experimental do Teorema de Bell, o qual afirma que a Mecânica Quântica não é uma teoria física realista local. Aqui, revisitamos a reformulação dada por N.D. Mermin em 1994 da proposta original de L. Hardy apresentada em 1992, definindo conceitos básicos, indicando realizações experimentais e discutindo tópicos relacionados.
\end{abstract}

Palavras-chave: Mecânica Quântica. Realismo Local. Experimentação.

Hardy's Experiment is an alternative to experimental verification of Bell's Theorem, which states that quantum mechanics is not a realistic local physical theory. Here we review the reformulation given by N.D. Mermin in 1994 of L. Hardy's original proposal presented in 1992, with the definition of basic concepts, indication of experimental achievements, and discussion of related topics.

Keywords: Quantum Mechanics. Local Realism. Experimentation.

\section{Introdução}

O Teorema de Bell [1] estabelece que a Mecânica Quântica não é uma teoria física realista local, fato corroborado por várias análises teóricas e também verificações experimentais. Por realismo local entendemos a conjunção destas duas condições:

- Realismo: os parâmetros mensuráveis de um sistema físico num estado puro possuem valores bemdefinidos em todos os instantes do tempo. Em particular, as medições não determinam valores para esses parâmetros, apenas os revela aos observadores.

- Localidade: os resultados da medida de um parâmetro físico não são afetados por quaisquer eventos ocorridos numa região causalmente disjunta da região em que a medida é realizada.

A demostração original do Teorema de Bell consiste na verificação de que as previsões da Mecânica Quântica violam as Desigualdades de Bell, um conjunto de desigualdades que devem ser satisfeitas pelas teorias físicas realistas locais [1] [2]. Posteriormente, foram apresentadas demonstrações que não envolvem desigualdades, dentre os quais destacam-se o Experimento GHZ [3] [4] (veja também [5]) e o Experimento de Hardy [6] [7]. Aqui, apresentamos e discutimos o Experimento de Hardy se-

*Endereço de correspondência: lucio.fassarella@ufes.br guindo ideias de Mermin 8].

O Experimento de Hardy, proposto em 6] 7], prova o Teorema de Bell numa situação que envolve apenas duas partículas 1. Essencialmente, o experimento consiste em uma fonte de pares de partículas que atravessam interferômetros de Mach-Zehnder e são medidas por detectores localizados em regiões causalmente disjuntas, numa configuração em que os detectores podem ser configurados em uma de duas alternativas de modo indepentende e aleatório.

A compreensão do experimento de Hardy é motivada pela sua simplicidade e beleza, além do que possui de instrutivo:

"Mas a importância adicional e a grande beleza do experimento de Hardy está no que ele nos diz, como uma dedução extremamente simples e direta da teoria quântica elementar, sobre o mundo que a teoria descreve na ausência de interações hipotéticas e não detectáveis que destroem correlações. (...) A brilhante refutação das hipóteses [subjacentes às derivações da desigualdade de Bell] permanece, em

${ }^{1} \mathrm{O}$ Experimento $G H Z$ envolve três partículas e o argumento original de Hardy também envolve três partículas; entretanto, enquanto no Experimento GHZ as três partículas são essenciais, no experimento discutido por Hardy com ternas elétron-pósitron-fóton, os fótons podem ser eliminados sem prejudicar o argumento. 
sua simplicidade básica, como uma das mais estranhas e bonitas pérolas que podemos encontrar no extraordinário solo da mecânica quântica." [8, tradução nossa]

A versão de Mermin, em comparação com a versão original do experimento de Hardy, possui o mérito de simplificar a descrição da situação experimental e do processo de medida pela eliminação de detalhes técnicos, bem como por reduzir os aspectos conceitual e lógico ao essencial - sendo por isso mais clara e objetiva, facilitando a compreensão do argumento. Em particular, Mermin não menciona absolutamente a ideia de variáveis ocultas, enquanto Hardy a inclui nos seus desenvolvimentos ${ }^{2}$

Essencialmente, o argumento de Hardy-Mermin consiste num reductio ad absurdum no qual a hipótese de realismo local implica numa contradição envolvendo os dados de uma situação experimental específica, que é realizável fisicamente e possui descrição mecânico quântica consistente.

$\mathrm{Na}$ sequência, apresentamos cada elemento desse argumento discutindo aspectos relevantes.

\section{Experimento de Hardy}

O experimento de Hardy possui um aparato experimental que consiste numa fonte $S$ de pares de partículas ${ }^{3} X$ e $Y$, e dois detectores, $D_{X}$ e $D_{Y}$ - conforme a Figura 1 Os pares de partículas são produzidos num estado específico $\left|\psi_{0}\right\rangle$. Cada detector possui duas configurações: $a$ ou $b$; em qualquer configuração, há duas alternativas para o resultado da medição: 0 ou 1. Para cada par de partículas produzido pela fonte, presumimos invariavelmente que (i) as configurações dos detectores são definidas após a emissão das partículas e (ii) a configuração do detector e a detecção de uma partícula são eventos causalmente disjuntos da configuração do detector e da detecção da outra partícula.

O experimento ocorre do seguinte modo: mediante acionamento pelo experimentador, a fonte emite um par de partículas e cada elemento do par é dirigido a um detector; entre o instante em que a fonte é acionada e o instante em que as partículas são detectadas, os detectores são configurados aleatória e independentemente em

${ }^{2} \mathrm{~A}$ ideia de variáveis ocultas também está na formulação original de Bell [1] e na discussão que a motivou, desenvolvida por Eintein, Podolsky e Rosen sobre a completude da Mecânica Quântica 9]:

"Numa teoria obtida da Mecânica Quântica pela introdução de parâmetros para determinar os resultados de medidas individuais sem modificação das predições estatísticas [viz., variáveis ocultas], deve haver um mecanismo pelo qual a preparação de instrumento de medida possa influenciar outro instrumento, independentemente da distância entre eles." 1, tradução nossa com ênfase acrescentada]

\footnotetext{
${ }^{3}$ As partículas podem ser idênticas ou não, mas são distinguíveis pelo menos quanto ao sentido em que são emitidas pela fonte.
}

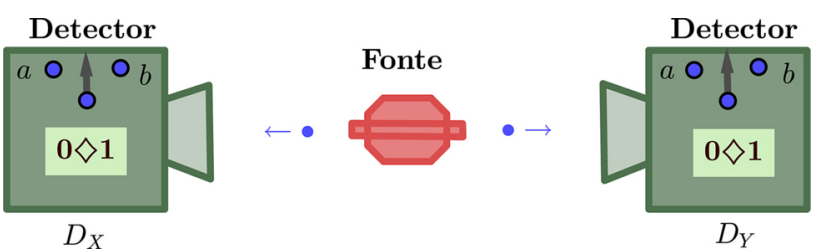

Figura 1: Esquema do Experimento de Hardy.

uma das duas alternativas, $a$ ou $b$; após as detecções, o resultado é registrado; essa sequência de eventos é repetida um número suficientemente grande de vezes.

Hipoteticamente, após realizar um número suficientemente grande de medições, os registros devem fornecer estimativas razoáveis para as probabilidades dos resultados possíveis, condicionadas às alternativas de configuração dos detectores. Baseados nessa hipótese, adotamos a seguinte notação para os experimentos e as probabilidades condicionais dos seus resultados (onde $u, v \in\{a, b\} \mathrm{e}$ $x, y \in\{0,1\})$ :

- $[x, y \mid u, v]$ designa um experimento em que os detectores $D_{X}$ e $D_{Y}$ são configurados nas alternativas $u$ e $v$ (respectivamente) e detectam os resultados $x$ e $y$ (respectivamente).

- $P[x, y \mid u, v]$ denota a probabilidade de um experimento $[x, y \mid u, v]$ :

$$
\begin{gathered}
P[x, y \mid u, v]= \\
\frac{\text { número de ocorrências do experimento }[x, y \mid u, v]}{\text { número total de experimentos realizados }} \text {. }
\end{gathered}
$$

Naturalmente, valem as seguintes condições:

$$
\left\{\begin{array}{l}
P[x, y \mid u, v] \geq 0, \\
\sum_{x^{\prime}, y^{\prime}=1,2} P\left[x^{\prime}, y^{\prime} \mid u, v\right]=1 .
\end{array}\right.
$$

Condições de Mermin. As condições de Mermin consistem destas três exigências sobre a ocorrência dos eventos no Experimento de Hardy, que podemos interpretar como condições características sobre a adequação da configuração do aparato experimental e do estado inicial dos pares de partícula:

1. nas medidas em que os detectores têm configurações diferentes, nunca ocorre de ambos detectarem o valor 0 :

$$
P[0,0 \mid a, b]=0=P[0,0 \mid b, a] ;
$$

2. nas medidas em que ambos detectores têm configuração $a$, nunca ocorre de ambos detectarem o valor 1:

$$
P[1,1 \mid a, a]=0 ;
$$

3. nas medidas em que ambos detectores têm configuração $b$, eventualmente ocorre de ambos detectarem o valor 0 :

$$
P[0,0 \mid b, b]>0
$$


Em princípio, poderia acontecer dessas condições serem teoricamente incompatíveis com a Mecânica Quântica ou delas serem experimentalmente impossíveis de realizar (ainda que sejam possíveis teoricamente). Contudo, mostraremos que elas são teoricamente possíveis e indicaremos realizações experimentais.

\section{O argumento de Hardy}

Suponha a validade da hipótese do realismo local no experimento de Hardy. Como o estado inicial dos pares de partícula está fixado pela fonte, o realismo implica que todos os possíveis eventos $[x, y \mid u, v]$ têm probabilidades de ocorrer pré-determinadas, independentemente das configurações dos detectores serem fixadas em algum momento entre a emissão e as detecções. Pela configuração do experimento, a localidade implica que o resultado da medidas de cada partícula de um par depende somente da configuração do seu respectivo detector, mas não da configuração do detector e do resultado da medida da outra partícula. Assim, considere um evento $[0,0 \mid b, b]$, que eventualmente ocorre devido à condição (5). Pela condição (3), deduzimos que se o detector $D_{X}$ estivesse na configuração $a$, então o resultado da medida da partícula $X$ teria sido necessariamente 1; analogamente, se o detector $D_{Y}$ estivesse na configuração $a$, o resultado da medida da partícula $Y$ também teria sido 1; agora, o realismo (novamente) nos permite concluir que se ambos detectores $D_{X}$ e $D_{Y}$ estivessem na configuração $a$, então as medidas das partículas $X$ e $Y$ deveriam ambas necessariamente resultar 1 ; ora, isso significa que $P[1,1 \mid a, a]>0 \sqrt{4}$ contudo, essa conclusão contradiz diretamente a condição de Mermin (4). Exatamente essa contradição nos permite concluir que a hipótese do realismo local não pode ser válida.

\section{Mecânica Quântica do experimento de Hardy}

Nesta seção mostro que a Mecânica Quântica é compatível com o experimento de Hardy, exibindo um modelo que satisfaz as condições de Mermin (3), (4), (5).

Considere um par de sistemas de dois níveis $\mathcal{S}_{1}$ e $\mathcal{S}_{2}$ acoplados, com espaço de Hilbert

$$
\mathcal{H}:=\mathcal{H}_{1} \otimes \mathcal{H}_{2} \text {. }
$$

Em cada sistema $\mathcal{S}_{j}(j=1$ ou 2$)$, escolhemos uma base de estados ortonormais em $\mathcal{H}_{j}$

$$
\left\{|a, 0\rangle_{j},|a, 1\rangle_{j}\right\}
$$

\footnotetext{
${ }^{4}$ No caso em que as configurações $a$ e $b$ dos detectores são equiprováveis, é possível concluir mais precisamente que $P[1,1 \mid a, a] \geq$ $P[0,0 \mid b, b]$.
}

bem como este outro par estados ortonormais: 5

$$
\begin{gathered}
|b, 0\rangle_{j}:=\frac{1}{\sqrt{2}}|a, 0\rangle_{j}+\frac{1}{\sqrt{2}}|a, 1\rangle_{j}, \\
|b, 1\rangle_{j}:=\frac{1}{\sqrt{2}}|a, 0\rangle_{j}-\frac{1}{\sqrt{2}}|a, 1\rangle_{j} ;(j=1 \text { ou } 2) .
\end{gathered}
$$

Para uso posterior, registramos as identidades:

$$
\begin{gathered}
\langle a, w \mid a, z\rangle_{j}=\delta_{w z},\langle b, w \mid b, z\rangle_{j}=\delta_{w z}, \\
\langle a, w \mid b, z\rangle_{j}=(-1)^{w z} \frac{1}{\sqrt{2}} ;(w, z=0,1 ; j=1,2) .
\end{gathered}
$$

Em cada sistema, definimos os observáveis

$$
\begin{gathered}
\hat{a}_{j}:=|a, 0\rangle_{j}\left\langle a,\left.0\right|_{j}+\mid a, 1\right\rangle_{j}\left\langle a,\left.1\right|_{j} \mathrm{e}\right. \\
\hat{b}_{j}:=|b, 0\rangle_{j}\left\langle b,\left.0\right|_{j}+\mid b, 1\right\rangle_{j}\left\langle b,\left.1\right|_{j}(j=1 \text { ou } 2) .\right.
\end{gathered}
$$

Para os estados do sistema composto, adotamos a seguinte notação:

$|u, x ; v, y\rangle:=|u, x\rangle_{1} \otimes|v, y\rangle_{2} ; u, v=a$ ou $b ; x, y=0$ ou 1

Dados $u \in\left\{a_{1}, b_{1}\right\}$ e $v \in\left\{a_{2}, b_{2}\right\}$, o operador $\hat{u} \otimes \hat{v}$ do sistema composto possui exatamente estes quatro autoestados ortonormalizados:

$$
|u, 0 ; v, 0\rangle,|u, 0 ; v, 1\rangle,|u, 1 ; v, 0\rangle,|u, 1 ; v, 1\rangle .
$$

Agora, considere um estado genérico do sistema composto:

$$
|\psi\rangle=\sum_{\xi, \zeta=0,1} c(\xi, \zeta)|a, \xi ; a, \zeta\rangle
$$

onde

$$
c(\xi, \zeta) \in \mathbb{C} \forall \xi, \eta \in\{0,1\}, \sum_{\xi, \zeta=0,1}|c(\xi, \zeta)|^{2}=1 .
$$

Para dados $u \in\left\{a_{1}, b_{1}\right\}$ e $v \in\left\{a_{2}, b_{2}\right\}$ e $x, y \in\{0,1\}$, a probabilidade de obtermos o resultado $(x, y)$ na medida do observável $\hat{u} \otimes \hat{v}$ do sistema composto quando este está no estado $|\psi\rangle$ é dada pela regra de Born:

$$
P[x, y \mid u, v]=|\langle u, x ; v, y|| \psi\rangle|^{2} .
$$

Vamos estabelecer as condições sobre os coeficientes $c(\xi, \zeta)$ para que as probabilidades cumpram as condições de Mermin (3), (4), (5). Partindo das identidades (6),

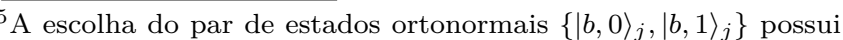
uma arbitrariedade limitada apenas pela condição de que esses estados possuam componentes não-nulas nas direções de ambos estados $|a, 0\rangle_{j}$ e $|a, 1\rangle_{j}$.
} 
calculamos:

$$
\begin{aligned}
P[0,0 \mid a, b] & =|\langle a, 0 ; b, 0 \mid \psi\rangle|^{2} \\
& =\left|\sum_{\xi, \zeta=0,1} c(\xi, \zeta)\langle a, 0 ; b, 0 \mid a, \xi ; a, \zeta\rangle\right|^{2} \\
& =\left.|\sum_{\xi, \zeta=0,1} c(\xi, \zeta) \underbrace{\langle a, 0 \mid a, \xi\rangle}_{=\delta_{0 \xi}} \underbrace{\langle b, 0| a, \zeta}_{=(-1)^{0 \zeta} / \sqrt{2}}\rangle\right|^{2} \\
& =\left|\frac{c(0,0)+c(0,1)}{2}\right|^{2},
\end{aligned}
$$

donde

$$
P[0,0 \mid a, b]=\frac{1}{2}|c(0,0)+c(0,1)|^{2} .
$$

Analogamente, calculamos:

$$
\begin{gathered}
P[0,0 \mid b, a]=\frac{1}{2}|c(0,0)+c(1,0)|^{2} ; \\
P[1,1 \mid a, a]=|c(1,1)|^{2} ; \\
P[0,0 \mid b, b]=\frac{1}{4}|c(0,0)+c(1,0)+c(0,1)+c(1,1)|^{2} .
\end{gathered}
$$

Assim, impondo as condições de Mermin (3), (4), (5) e a condição de normalização (8) à essas probabilidades, temos o sistema:

$$
\left\{\begin{array}{l}
|c(0,0)+c(0,1)|^{2}=0 \\
|c(0,0)+c(1,0)|^{2}=0 \\
|c(1,1)|^{2}=0 \\
|c(0,0)+c(1,0)+c(0,1)+c(1,1)|^{2}>0 \\
\sum_{\xi, \zeta=0,1}|c(\xi, \zeta)|^{2}=1
\end{array}\right.
$$

A única solução desse sistema $\hat{\epsilon}^{6}$

$$
c(1,1)=0, c(0,0)=-c(0,1)=-c(1,0)=\frac{1}{\sqrt{3}} .
$$

Sumariando, o sistema composto pode ser preparado para realizar o experimento de Hardy com o detector da componente $\mathcal{S}_{1}$ configurado para medir os observáveis $\hat{a}_{1}$ ou $\hat{b}_{1}$, o detector da componente $\mathcal{S}_{2}$ configurado para medir os observáveis $\hat{a}_{2}$ ou $\hat{b}_{2}$ e o estado do sistema composto preparado nesta superposição emaranhada:

$$
\left|\psi_{0}\right\rangle=\frac{1}{\sqrt{3}}|a, 0 ; a, 0\rangle-\frac{1}{\sqrt{3}}|a, 0 ; a, 1\rangle-\frac{1}{\sqrt{3}}|a, 1 ; a, 0\rangle .
$$

\section{Realizações do experimento de Hardy}

O artigo original de Hardy 6] descreve a situação específica em que o aparato experimental é constituído por dois interferômetros de Mach-Zehnder dispostos de modo que as trajetórias das partículas de pares elétron-pósitron se cruzem, permitindo-as interagir aniquilando-se ou não. Num artigo posterior [7], ele estendeu sua análise e mostrou que

\footnotetext{
${ }^{6}$ Nesse caso específico, temos $P[0,0 \mid b, b]=1 / 12 \approx 0.083$ - valor compatível com o máximo possível para esse sistema, que é $1 / \tau^{5}$, sendo $\tau=(1+\sqrt{5}) / 2$ a razão áurea 8 .
}

"é possível demonstrar a não-localidade para duas partículas sem o uso de desigualdades para todos os estados emaranhados exceto aqueles maximalmente emaranhados, tais como os estados singletos. Os autovetores correspondentes às medidas que devem ser realizadas para fazer isso [...] têm uma relação particularmente simples com o estado emaranhado."

Essa proposta foi realizada pouco tempo depois por Torgerson, Branning, Monken e Mandel medindo polarizações em pares de fótons 10. Experimentos similares e refinamentos foram realizados por outros grupos, e.g. 11], 12], 13 .

Existem generalizações do Experimento de Hardy e correspondentes realizações experimentais (e.g., [14] e [15]), as quais podem contradizer ainda mais contundentemente o realismo local:7

"Nós realizamos o primeiro teste experimental da não-localidade quântica via uma generalização do paradoxo de Hardy [...]. Teoreticamente, provou-se que para qualquer $n \geq 3$, o paradoxo de Hardy é sempre mais forte que aqueles previamente conhecidos.". 15, com tradução nossa].

\section{Algumas considerações}

O experimento original de Hardy possui a peculiaridade de permitir a interação de uma partícula fermiônica com sua antipartícula sem que o par seja necessariamente aniquilado (só eventualmente) - fenômeno chamado na literatura de Paradoxo de Hardy. Variações do experimento podem não exibir esse fenômeno, particularmente no caso das medidas incidirem sobre partículas bosônicas (e.g., fótons).

O experimento de Hardy requer uma fonte de informação randômica para poder ser realizado, circunstância compartilhada com os testes da desigualdade de Bell 16 . Capítulo 7]. Entretanto, qualquer dispositivo empregado como fonte deixa em aberto a possibilidade dele ser determinado por variáveis ocultas - o que constitui a chamada lacuna da liberdade de escolha [freedom-of-choice loophole]. Foram apresentadas propostas para contornar esse problema, dentre as quais usar dados obtidos por observações dos limites do universo e escolhas humanas 17] [16]. Dentre todos os experimentos realizados até agora com a intenção de contornar a lacuna da liberdadede-escolha cabe destacar o BIG Bell Test, "um conjunto

${ }^{7}$ Explico o termo paradoxo de Hardy na próxima seção, mas a citação pode ser bem compreendida interpretando o termo como uma classe particular de experimentos de Hardy. 
de testes do realismo local usando escolhas humanas, o que evita suposições sobre a previsibilidade na física" [18, assim descrito pelos seus responsáveis:

"Recrutamos cerca de 100.000 participantes humanos para jogar um videogame on-line que incentiva a entrada rápida e sustentada de seleções imprevisíveis e ilustra a metodologia do teste Bell. Os participantes geraram 97.347.490 escolhas binárias, que foram direcionadas através de uma plataforma web escalável para 12 laboratórios em cinco continentes, onde 13 experimentos testaram o realismo local usando fótons, átomos isolados, conjuntos atômicos e dispositivos supercondutores. Em um período de 12 horas em 30 de novembro de 2016, participantes no mundo todo forneceram um fluxo de dados sustentado de mais de 1.000 bits por segundo para os experimentos, que usaram os diferentes dados gerados por humanos para escolher cada configuração de medição. As correlações observadas contradizem fortemente o realismo local e outras posições realistas em cenários bipartidos e tripartidos. Os resultados do projeto incluem o fechamento da 'lacuna da liberdade de escolha' [freedom-of-choice loophole] (a possibilidade de que as escolhas de configuração sejam influenciadas por 'variáveis ocultas' para se correlacionarem com as propriedades das partículas), a utilização dos métodos de videogame para coleta rápida de aleatoriedade gerada pelo homem e o uso de técnicas de rede para participação global em ciência experimental." 19, com tradução nossa]

Existem diversas críticas e críticas de críticas aos teoremas e experimentos que refutam o realismo local da Mecânica Quântica. Uma crítica que atinge o experimento de Hardy foca o fato do argumento ser do tipo contrafactual, ou seja, dele recorrer à análise de supostos resultados de medidas que seriam observadas caso algumas variáveis do experimento tivessem sido diferentes (viz., as configurações dos detectores):

"O teorema de Bell, bem como suas variantes de GHZ e de Hardy não invalidam o realismo local e que as correlações encontradas nas experiências, que visam informar estes teoremas, nada têm a ver com a nãolocalidade, sendo meramente, correlações realistas locais, perfeitamente explicáveis, tanto quando os argumentos contrafatuais são eliminados dos pressupostos destes teoremas, como em termos de um efeito clássico puramente topológico." 18 .
Aqui a discussão torna-se sutil e extrapola nossa intenção para este trabalho, pelo que nos limitamos a indicar a leitura do artigo [20].

\section{Agradecimentos}

Agradeço aos colegas do Departamento de Matemática Aplicada e demais professores da Universidade Federal do Espírito Santo pela concessão de uma licença por três meses para estudos programados, pela qual pude me dedicar minha atenção aos fundamentos da Mecânica Quântica e iniciar a redação deste artigo.

\section{Referências}

[1] J.S. Bell, Physics 1, 195 (1964).

[2] J. Pinochet e D. Rojas-Líbano, Rev. Bras. Ens. Fis. 38, e3303 (2016).

[3] D.M. Greenberger, M.A. Horne e A. Zeilinger, in Bells Theorem, Quantum Theory and Conceptions of the Universe editado por M. Kafatos (Kluwer, Dordrecht, 1989).

[4] D.M. Greenberger, M.A. Horne, A. Shimony e A. Zeilinger, Am. J. Phys. 58, 1131 (1990).

[5] N.D. Mermin, Phys. Rev. Lett. 65, 3373 (1990).

[6] L. Hardy, Phys. Rev. Lett. 68, 2981 (1992).

[7] L. Hardy, Physical Review Letters 71, 1665 (1993).

[8] N.D. Mermin, Am. J. Phys. 62, 880 (1994).

[9] A. Einstein, B. Podolsky e N. Rosen, Physical Review 47, 777 (1935)

[10] J.R. Torgerson, D. Branning, C.H. Monken e L. Mandel, Phys. Lett. A 204, 323 (1995)

[11] G. Di Giuseppe, F. De Martini e D. Boschi, Phys. Rev. A 56176 (1997).

[12] D. Boschi, S. Branca, F. De Martini e L. Hardy, Phys. Rev. Lett. 792755 (1997).

[13] W.T.M. Irvine, J.F. Hodelin, C. Simon e D. Bouwmeester, Physical Review Letters 95, 030401 (2005).

[14] S.H. Jiang, Z.P. Xu, H.Y. Su, A.K. Pati e J.L. Chen, Physical Review Letters 120, 050403 (2018).

[15] Y.H. Luo, H.Y. Su, H.L. Huang, X.L. Wang, T. Yang, L. Li, N.L. Liu, J.L. Chen, C.Y. Lu e J.W. Pan, Science Bulletin 631611 (2018).

[16] J.S. Bell, Speakable and Unspeakable in Quantum Mechanics (Cambridge University Press, Cambridge, 2004).

[17] Y. Aharonov, A. Botero, S. Popescu, B. Reznik e J. Tollaksen, Physics Letters A 301, 130 (2002).

[18] Á. Balsas, Realismo e Localidade em Mecânica Quântica (Editora Livraria da Física, São Paulo, 2013).

[19] The BIG Bell Test Collaboration, Nature 557, 212 (2018).

[20] Y. Aharonov, A. Botero, S. Popescu, B. Reznik e J. Tollaksen, Physics Letters A 301, 130 (2002). 